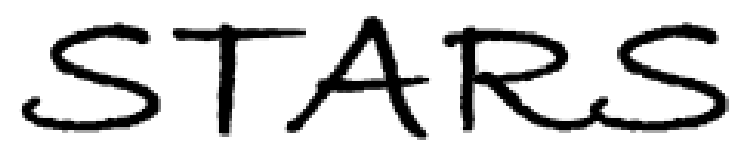

University of Central Florida

STARS

Faculty Bibliography 2000s

Faculty Bibliography

$1-1-2004$

\title{
Dual-frequency addressed hybrid-aligned nematic liquid crystal
}

Yan-Qing Lu

University of Central Florida

Xiao Liang

University of Central Florida

Yung-Hsun Wu

University of Central Florida

Fang Du

University of Central Florida

Shin-Tson Wu

University of Central Florida

Find similar works at: https://stars.library.ucf.edu/facultybib2000

University of Central Florida Libraries http://library.ucf.edu

This Article is brought to you for free and open access by the Faculty Bibliography at STARS. It has been accepted for inclusion in Faculty Bibliography 2000 s by an authorized administrator of STARS. For more information, please contact STARS@ucf.edu.

\section{Recommended Citation}

Lu, Yan-Qing; Liang, Xiao; Wu, Yung-Hsun; Du, Fang; and Wu, Shin-Tson, "Dual-frequency addressed hybridaligned nematic liquid crystal" (2004). Faculty Bibliography 2000s. 4564.

https://stars.library.ucf.edu/facultybib2000/4564

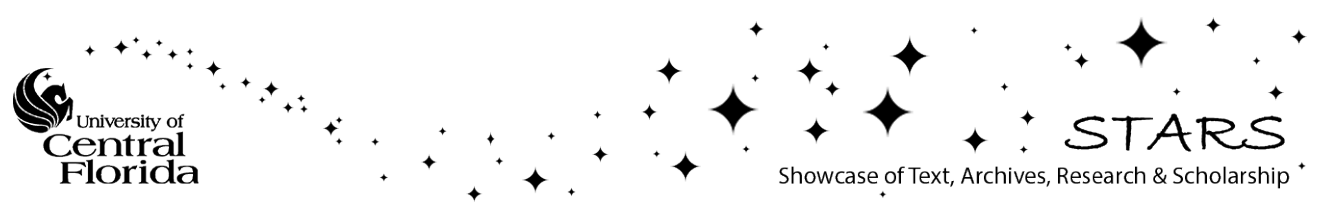




\section{Dual-frequency addressed hybrid-aligned nematic liquid crystal}

Cite as: Appl. Phys. Lett. 85, 3354 (2004); https://doi.org/10.1063/1.1809282

Submitted: 06 July 2004 . Accepted: 27 August 2004 . Published Online: 22 October 2004

Yan-Qing Lu, Xiao Liang, Yung-Hsun Wu, Fang Du, and Shin-Tson Wu

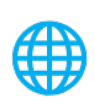

\section{ARTICLES YOU MAY BE INTERESTED IN}

Fast switching dual-frequency liquid crystal optical retarder, driven by an amplitude and frequency modulated voltage

Applied Physics Letters 83, 3864 (2003); https://doi.org/10.1063/1.1625114

Dielectric heating effects of dual-frequency liquid crystals

Applied Physics Letters 86, 231104 (2005); https://doi.org/10.1063/1.1944889

Field-induced deformation of hybrid-aligned nematic liquid crystals: New multicolor liquid crystal display

Journal of Applied Physics 47, 3842 (1976); https://doi.org/10.1063/1.323245

\section{Applied Physics Letters}

Mid-IR and THz frequency combs special collection

\section{Read Now!}




\title{
Dual-frequency addressed hybrid-aligned nematic liquid crystal
}

\author{
Yan-Qing Lu, Xiao Liang, Yung-Hsun Wu, Fang Du, and Shin-Tson Wu ${ }^{\text {a) }}$ \\ College of Optics and Photonics, University of Central Florida, Orlando, Florida 32816
}

(Received 6 July 2004; accepted 27 August 2004)

\begin{abstract}
Dual-frequency addressed hybrid-aligned nematic (HAN) liquid crystal cell is demonstrated as a variable optical attenuator at $1.55 \mu \mathrm{m}$ wavelength. By controlling the low- and high-frequency electric field, the dual-frequency liquid crystal (DFLC) molecules can be reoriented parallel or perpendicular to the substrates so that the maximum obtainable phase modulation is doubled. In comparison to a homogeneous cell, the DFLC HAN cell shows a lower operating voltage and faster response time. Furthermore, the DFLC HAN cell exhibits three stable states that have some applications such as ternary photonic devices. () 2004 American Institute of Physics.
\end{abstract}

[DOI: 10.1063/1.1809282]

Liquid crystal (LC) has been used extensively for information display, ${ }^{1}$ laser beam steering, ${ }^{2}$ spatial light modulator, ${ }^{3}$ tunable photonic crystal, ${ }^{4}$ and variable optical attenuator (VOA). ${ }^{5}$ For intensity modulation, twisted nematic $^{6}$ and homeotropic ${ }^{7}$ cells are commonly used because of their high contrast ratio and low operation voltage. However, for phase modulation, thin homogeneous cell is a favored choice because of fast response time and low operating voltage. ${ }^{8}$ To obtain submillisecond response time, stressed LC, ${ }^{9}$ polymer-network LC, ${ }^{10}$ and high-pretilt dual-frequency $\mathrm{LC}^{11}$ have been proposed. A common drawback of these approaches is that the operating voltage is quite high ( $\sim 100 \mathrm{~V}_{\text {rms }}$ ). There is an urgent need to reduce the LC operating voltage for high speed LC phase modulators.

Hybrid aligned nematic (HAN) cell ${ }^{12}$ is known to have no threshold voltage. In a HAN cell, as shown in Fig. 1(a), the top substrate has homogeneous alignment while the bottom substrate has homeotropic alignment. Due to this special molecular configuration, the Freederisckz transition threshold no longer exists. It is therefore possible to achieve grayscales with low voltage which is very beneficial for displays and photonics applications. ${ }^{13}$ However, a shortcoming of the HAN cell is that its phase retardation is only about one half of that of a corresponding homogeneous or homeotropic cell. For a LC optical phased array, a $2 \pi$ phase change is required. For a transmission type VOA, there should be at least a $\pi$ phase tuning range. The reduced phase change of the HAN cell limits its applications, especially for the photonic applications in the infrared region.

In this letter, we present a dual-frequency liquid crystal $\left(\right.$ DFLC) ${ }^{14}$ HAN cell for photonic applications with emphasis on VOA at $\lambda=1.5 \mu \mathrm{m}$. We demonstrate theoretically and experimentally that the dual-frequency electric fields can effectively drive the LC molecules to either parallel or perpendicular position with respect to the substrates. The total obtainable phase change is equivalent to that of a homogeneous cell but at a lower voltage. Furthermore, the optical decay time is Five times faster than that using singlefrequency addressing. Finally, the DFLC HAN cell has three stable states at high frequency, low frequency, and zero field, respectively, some ternary optical devices are also proposed.

\footnotetext{
${ }^{\text {a)} E l e c t r o n i c ~ m a i l: ~ s w u @ ~ @ a i l . u c f . e d u ~}$
}

When there is no external field across a HAN cell, the LC directors vary smoothly from a vertical state to a horizontal state, as shown in Fig. 1(a), due to the different surface treatment. Using the Frank elasticity theory, assuming a strong anchoring at both substrates and using the single elastic constant approximation $\left(k=k_{11}=k_{22}=k_{33}\right)$, we obtain the initial elastic director orientation angle $\phi_{0}(z) \approx \pi z / 2 d,{ }^{13}$ which is approximately a linear function of the LC layer depth $(z)$, assuming the cell gap $(d)$ is along the $z$ axis. The corresponding phase retardation $\Gamma$ of this cell can be expressed as

$$
\Gamma=\frac{2 \pi}{\lambda} n_{o} d\left[\frac{1}{d} \int_{0}^{d} \frac{d z}{\left(1-R \sin ^{2} \phi\right)^{1 / 2}}-1\right],
$$

where $R \equiv 1-n_{o}^{2} / n_{e}^{2} ; n_{e}$ and $n_{o}$ are the extraordinary and ordinary refractive indices, respectively; $\lambda$ is the wavelength; $\phi$ is the LC director orientation angle which equals $\phi_{o}$ in the field-off state. Although the actual $\Gamma$ value is determined by the ratio between $n_{o}$ and $n_{e}$, it can be proved that the phase retardation of a HAN cell is almost half of that a homogeneous (or homeotropic) cell, provided that the same LC material and cell gap are used. This is because the hybrid molecular alignment where the LCs near the bottom substrate (see Fig. 1) makes smaller contribution to the observed phase change.

When a low-frequency electric field $E$ is applied, the LC directors tend to follow the electric field so that the director orientation angle $\phi(z)$ is changed. The equilibrium alignment in this field-on case can be determined by minimizing the total free energy

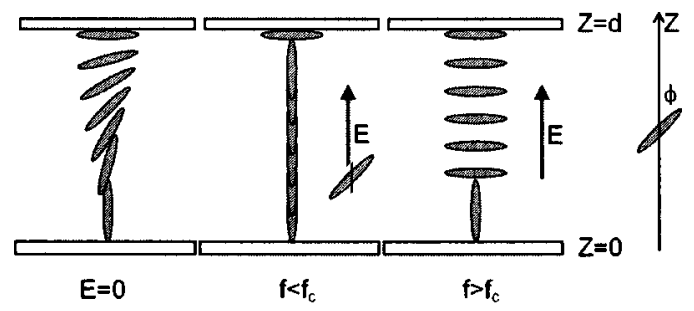

(a)

(b)

(c)

FIG. 1. Schematics of a HAN cell. The rods represent the LC molecules with the director orientation of $\phi(z)$. (a) Initial state at $V=0$; (b) a high voltage state at $f<f_{c}$; (c) a high voltage state at $f>f_{c}$. 


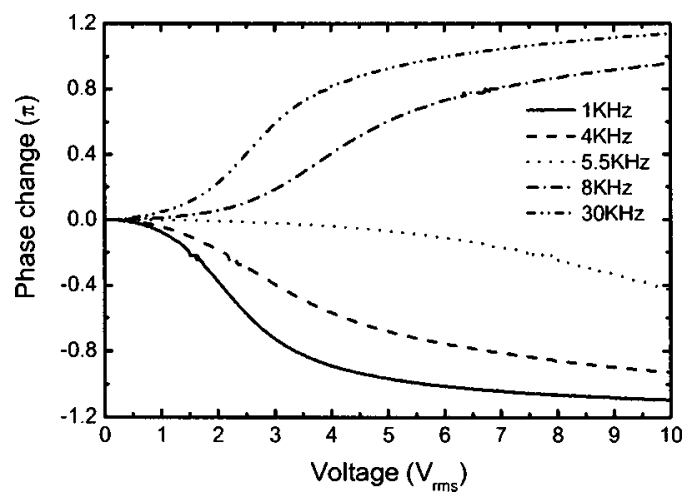

FIG. 2. The measured phase change of an $8.5 \mu \mathrm{m}$ HAN cell as a function of the applied voltage under different driving frequencies. From bottom to top, $f=1,4,5.5,8$, and $30 \mathrm{kHz}$.

$$
F=\frac{1}{2} \int_{0}^{d}\left\{k\left[\frac{\partial \phi(z)}{\partial z}\right]^{2}-\Delta \varepsilon E^{2} \cos ^{2} \phi(z)\right\} d z,
$$

where $\triangle \varepsilon=\varepsilon_{/ /}-\varepsilon_{\perp}$ is dielectric anisotropy, defined by the difference of dielectric constant parallel and perpendicular to the LC director. Assuming a small molecular deformation, the director orientation angle change is given by

$$
\phi(z)-\phi_{0}(z)=-\frac{\Delta \varepsilon E^{2}}{2 k}\left(\frac{d}{\pi}\right)^{2} \sin \left(\frac{\pi z}{d}\right)
$$

which is directly proportional to $E^{2}$ and shows no threshold. However, for a DFLC mixture the $\Delta \varepsilon$ is dependent on the driving frequency $(f) .{ }^{14}$ When the frequency is below crossover frequency, i.e., $f<f_{c}, \Delta \varepsilon$ is positive, but gradually changes to negative when $f>f_{c}$. In the high frequency regime, the DFLC behaves likes a negative $\Delta \varepsilon$ LC. Thus, the dielectric anisotropy $\Delta \varepsilon(f)$ in Eq. (2) is frequency dependent. As a consequence, $\phi(z)$ and $\Gamma$ are all frequency dependent for a DFLC filled HAN cell.

To realize the frequency tuning in a HAN cell as predicted above, an $8.5 \mu \mathrm{m}$ HAN cell filled with our homemade DFLC is used for the experiments. The physical properties of our DFLC mixture at room temperature $\left(T=25^{\circ} \mathrm{C}\right)$ are summarized as follows: crossover frequency $f_{c} \approx 6 \mathrm{kHz}, \Delta n=n_{e}$ $-n_{o}=0.24$ at $\lambda=1.55 \mu \mathrm{m}$, and $\Delta \varepsilon=+4.7$ at $f=1 \mathrm{kHz}$ and $\Delta \varepsilon=-3.9$ at $f=30 \mathrm{kHz}$. To measure the phase retardation, the DFLC HAN cell was placed between a pair of crossed polarizers. ${ }^{15}$ An Ando AQ4321D tunable laser operating at $1.55 \mu \mathrm{m}$ served as the light source. A computer controlled LabVIEW system was used for data recording and processing.

Figure 2 shows the measured phase modulation under different driving frequencies. The solid curve is for $f$ $=1 \mathrm{kHz}$. Because of the positive $\Delta \varepsilon$, in the voltage-on state the LC molecules are reoriented along the field direction as described in Fig. 1(b). The phase retardation thus decreases. When the frequency is elevated to $4 \mathrm{kHz}$, the phase retardation follows the similar trend but the phase modulation depth decreases due to the smaller $|\Delta \varepsilon|$. As $f \sim f_{c}$ which is $\sim 6 \mathrm{kHz}$, the LC directors are hardly reoriented. However, when $f>f_{c}, \Delta \varepsilon$ changes sign and becomes negative. The LC directors tend to be perpendicular to the electric field, as shown in Fig. 1(c). In this case, the phase retardation increases with the applied voltage. The results of $f=8 \mathrm{kHz}$ and $f=30 \mathrm{kHz}$ are illustrated in Fig. 2.

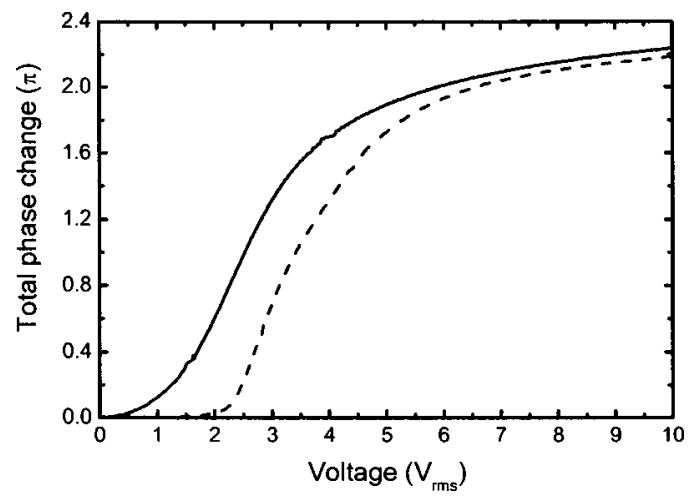

FIG. 3. The total achievable phase changes of two $8.5 \mu \mathrm{m} \mathrm{LC}$ cells in different alignments. The solid line is for the HAN cell, while the dashed lines are for a homogeneous cell.

From Fig. 2, both voltage and frequency can be used to tune the DFLC HAN cell to a desired retardation state, which makes the active driving between two arbitrary states possible. To obtain a larger phase change, the cell normally works at frequencies far from the crossover frequency. In our DFLC mixture, our low and high frequencies are chosen to be $f=1$ and $30 \mathrm{kHz}$, respectively. From Fig. 2, if we apply a $1 \mathrm{kHz}, 10 \mathrm{~V}_{\text {rms }}$ voltage to the HAN cell, the maximum obtainable phase is $-1.15 \pi$ at $\lambda=1.55 \mu \mathrm{m}$. This value is about $50 \%$ of the total phase retardation of an $8.5-\mu \mathrm{m}$-thick homogeneous LC cell, which is $2.63 \pi$. However, to obtain the maximum phase change of the DFLC HAN cell, we could keep voltage at $V=10 \mathrm{~V}_{\text {rms }}$ but change the frequency from 1 to $30 \mathrm{kHz}$. The total phase change is thus doubled, which is one of the main advantages of the DFLC HAN cell.

To compare the DFLC HAN cell with the homogeneous cell for phase modulation, Fig. 3 displays their voltagedependent phase changes. The cell gap and the employed LC material are the same. The HAN cell is driven between 1 and $30 \mathrm{kHz}$, but the homogeneous cell is driven by a $1 \mathrm{kHz}$ electric field. From Fig. 3, the HAN cell has no threshold voltage while the homogeneous cell has $V_{\text {th }} \sim 2 \mathrm{~V}_{\text {rms }}$. Therefore, to achieve the same phase change (say, $1 \pi$ ) the HAN cell has a lower operating voltage than the corresponding homogeneous cell.

Besides the lower operation voltage, the dual-frequency HAN cell also exhibits a much faster response time than the corresponding homogeneous cell. In a homogeneous cell, the rise time can be greatly improved by using the overdrive scheme. ${ }^{15}$ However, the decay time relies on the elastic recovery of LC molecules; it is determined by the visco-elastic coefficient of the LC material and the cell gap. ${ }^{1}$ To improve response time, polymer network liquid crystal has been considered. However, the high operating voltage and light scattering become serious concerns. ${ }^{10}$

The DFLC HAN cell offers an advantage for improving response time. In both rise and decay periods, the electric field is present. As a result, both rise and decay time can be improved significantly. Figure 4 shows the optical decay time of a DFLC HAN cell based zero-threshold VOA working at $1.55 \mu \mathrm{m}$. A compensation film is employed so that the VOA is in the bright state at $V=0$. When a $5.8 \mathrm{~V}_{\text {rms }} 1 \mathrm{kHz}$ signal is applied, the VOA is in its $-\pi$ state which corresponds to the "OFF" state. At this time, if the field is removed the VOA changes from its OFF state to ON state. The transition time ( $10 \%$ to $90 \%$ intensity) is $\sim 90 \mathrm{~ms}$, as shown 


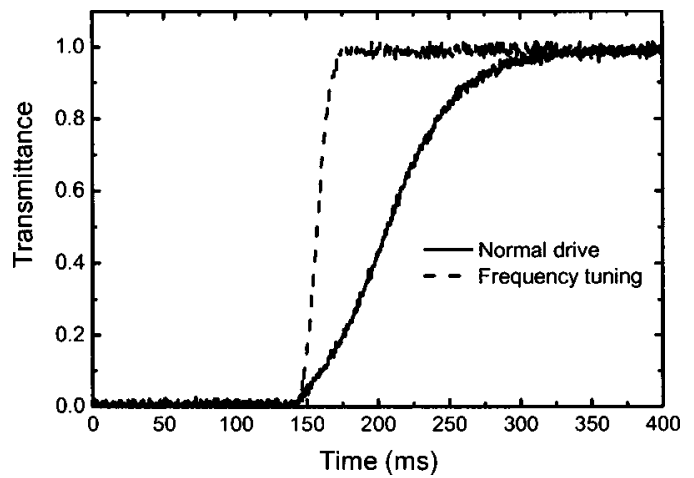

FIG. 4. The optical decay time of an $8.5 \mu \mathrm{m}$ DFLC HAN cell based VOA. The solid line is the normal decay curve by instantly turning off the $5.8 \mathrm{~V}_{\text {rms }}$ driving voltage. The dashed lines are for the dual frequency driving, where a $35 \mathrm{~ms}, 30 \mathrm{kHz}$ voltage burst is used to assist the LC directors relaxation.

by the solid line in Fig. 4. This is to mimic conventional single frequency driving in a HAN cell. However, because of the dual frequency addressing of the DFLC HAN cell, we keep voltage on the cell but switch frequency from 1 to $30 \mathrm{kHz}$ for $35 \mathrm{~ms}$ before turning off the voltage. The highfrequency electric field drives the LC molecules back. Thus, the optical decay time is shortened to $\sim 18 \mathrm{~ms}$, as shown by the dashed lines in Fig. 4.

Similarly, we could drive the LC to $+\pi$ phase state by applying a $6 \mathrm{~V}_{\mathrm{rms}} 30 \mathrm{kHz}$ field, then keep the voltage but decrease the frequency to $f=1 \mathrm{kHz}$ to tune the VOA back to its $\mathrm{ON}$ state. We find that the response time is also remarkably improved, which means that the frequency tuning is really a simple and effective approach to accelerate HAN cell's optical response without scattering. To further improve the response time, the overdrive and undershoot scheme can be employed and a submillisecond response time can be achieved. The tradeoff is in need of a short and high overdrive voltage. $^{11,16}$

A special feature of the above-mentioned VOA is that it has two stable "OFF" states: $-\pi$ phase and $\pi$ phase for the low- and high-frequency driving, respectively. Although it has binary amplitude $(0,1)$ states, it has ternary discrete phase $(-1,0,+1$ or $0,1,2)$ states. The DFLC HAN cell thus supplies a simple tri-stable system in high-frequency, lowfrequency, and no-field states, respectively. Ternary system is nature's most efficient numbering scheme; it has attracted a lot of attention in different disciplines. The ternary states in LC materials and devices have been explored. ${ }^{17,18}$ However, these approaches are either too complicated or not stable enough, while our DFLC addressed HAN cell could generate three stable states easily. Some applications, e.g., the ternary phase modulator, ternary logic device, and positive and negative tunable LC lens, by using the three stable states of the DFLC HAN cell are expected.

In conclusion, a dual-frequency addressed HAN cell for VOA application is demonstrated. The VOA shows no threshold voltage and a much faster response time. In comparison with the conventional single-frequency addressed HAN cell, the dual-frequency HAN cell has doubled phase retardation. Because of the unique tri-stability feature of the DFLC HAN cell, some applications such as the ternary optical devices are suggested.

This work is supported by AFOSR under Contact No. F49620-01-1-0377.

${ }^{1}$ S. T. Wu and D. K. Yang, Reflective Liquid Crystal Displays (Wiley, New York, 2001)

${ }^{2}$ R. Ozaki, Y. Matsuhisa, M. Ozaki, and K. Yoshino, Appl. Phys. Lett. 84, 1844 (2004).

${ }^{3}$ U. Efron, Spatial Light Modulator Technology (Dekker, New York, 1995).

${ }^{4}$ P. F. McManamon, T. A. Dorschner, D. L. Corkum, L. Friedman, D. S. Hobbs, M. Holz, S. Liberman, H. Q. Nguyen, D. P. Resler, R. C. Sharp, and E. A. Watson, Proc. IEEE 84, 268 (1996).

${ }^{5}$ C. Mao, M. Xu, W. Feng, T. Huang, K. Wu, and J. Liu, Proc. SPIE 5003, 121 (2003).

${ }^{6}$ M. Schadt and W. Helfrich, Appl. Phys. Lett. 18, 127 (1971).

${ }^{7}$ M. F. Schiekel and K. Fahrenschon, Appl. Phys. Lett. 19, 391 (1971).

${ }^{8}$ S. T. Wu and U. Efron, Appl. Phys. Lett. 48, 624 (1986).

${ }^{9}$ J. L. West, G. Zhang, and A. Glushchenko, SID Tech. Digest 34, 1469 (2003).

${ }^{10}$ Y. H. Fan, Y. H. Lin, H. Ren, and S. T. Wu, Appl. Phys. Lett. 84, 1233 (2004).

${ }^{11}$ A. B. Golovin, S. V. Shiyanovskii, and O. D. Lavrentovich, Appl. Phys. Lett. 83, 3864 (2003).

${ }^{12}$ S. Matsumoto, M. Kawamoto, and K. Mizunoya, J. Appl. Phys. 47, 3842 (1976).

${ }^{13}$ J. P. Drolet, J. S. Patel, K. G. Haritos, W. Xu, A. Scherer, and D. Psaltis, Opt. Lett. 20, 2222 (1995).

${ }^{14}$ H. K. Bucher, R. T. Klingbiel, and J. P. VanMeter, Appl. Phys. Lett. 25, 186 (1974).

${ }^{15}$ S. T. Wu, U. Efron, and L. D. Hess, Appl. Opt. 23, 3911 (1984).

${ }^{16}$ S. T. Wu, Appl. Phys. Lett. 57, 986 (1990).

${ }^{17}$ A. Au, C. S. Wu, S. T. Wu, and U. Efron, Appl. Opt. 34, 281 (1995).

${ }^{18}$ J. H. Kim, M. Yoneya, and H. Yokoyama, Nature (London) 420, 159 (2002). 